\title{
A rare variant of inguinal hernia: cryptorchid testis at the age of 60: an unusual case report
}

\begin{abstract}
Cryptorchidism is characterized by the extra-scrotal position of the testis. Although inguinal hernia is frequent, cryptorchid testis at the age of 60years is an uncommon entity. We wish to present this extremely rare case of inguinal hernia, which is the first one that we have encountered in our practice, along with the accompanying prognosis and therapeutic issues and a review of the literature.
\end{abstract}

Keywords: Cryptorchidism, extra-scrotal position, inguinal hernias

\author{
Volume 6 Issue 4 - 2017
}

Sachin Pathak,' Rajurkar Apurva Ajit, ${ }^{2}$ Sapan Sanjivkumar Parikh, ${ }^{2}$ Nirmal Ramsinhbhai Barad, ${ }^{2}$ Mehulkumar Prabhudas Kubavat, ${ }^{2}$ Abhishek Singh Nayyar ${ }^{3}$

'Department of General Surgery, Saraswati-Dhanwantari Dental College and Hospital and Post-Graduate Research Institute, India

${ }^{2}$ Department of Anesthesiology, BJ Medical College, India ${ }^{3}$ Department of Oral and Maxillo-facial Medicine and Radiology, Saraswati-Dhanwantari Dental College and Hospital and PostGraduate Research Institute, India

Correspondence: Abhishek Singh Nayyar, Department of Oral Medicine and Radiology, Saraswati-Dhanwantari Dental College and Hospital and Post-Graduate Research Institute, India, Tel +9|-98509 04067, Email singhabhishekndls@gmail.com

Received: April 6, 2017 | Published: April 13,2017

\section{Introduction}

Cryptorchidism is characterized by the extra-scrotal position of the testis. Cryptorchidism in a 60years old adult male is rare. Although inguinal hernia is frequent, cryptorchid testis at the age of 60years is an uncommon entity. The cryptorchid testis is characterized by the extrascrotal position of the testis. Longui $\mathrm{CA}^{1}$ this case report demonstrates the utility to understand the surgical anatomy of inguinal hernias. We wish to present this extremely rare case of inguinal hernia, which is the first one that we have encountered in our practice, along with the accompanying prognosis and therapeutic issues and a review of the literature.

\section{Case report}

We report the case of a 60years old adult male patient who was admitted due to left inguinal hernia. His medical history was not found to be relevant. Patient was referred with an inguinal hernia with recurrent severe colicky pain on appearance of hernia. When the patient used to cough, the hernia used to appear. Physical examination revealed left inguinal hernia. The scrotum showed absence of left testis in the pre-operative period (Figure 1). The diagnosis was left inguinal hernia with absent left testis. The rest of the examination was unremarkable. He had no known history of testicular disease. The patient did not have a problem of infertility. Surgical exploration revealed the presence of cryptorchid testicle in inguinal hernia. The left testis with its overlying tunica vaginalis was found at the deep inguinal ring. A viable normal sized testis was found in the hernial sac with its blood vessels and vas deferens. The testicle was viable. Left testicle was resected for the fear of malignancy. The hernia sac was, also, resected (Figure 2-5). Surgical correction was made and the hernia was repaired using a synthetic mesh (Lichtenstein Hernioplasty) (Figure 6) (Figure 7). Histopathological examination confirmed a normal testis and the presence of leydig cells and the seminiferous tubule without testicular germ cell tumor (TGCT). The patient tolerated the procedure well and the post-operative period was uneventful.

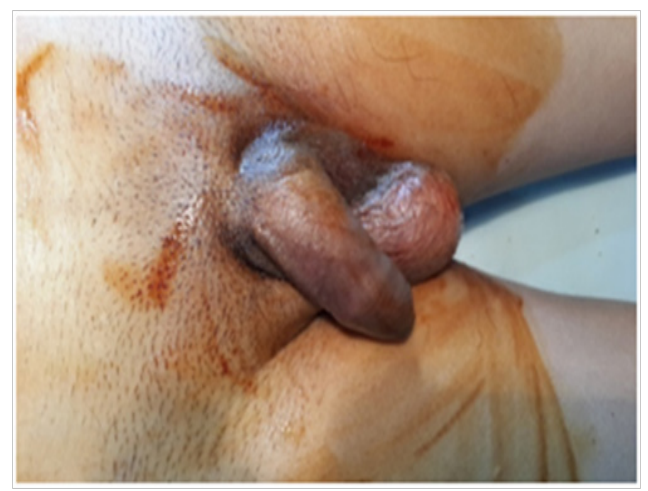

Figure I Congenitally absent left testis.

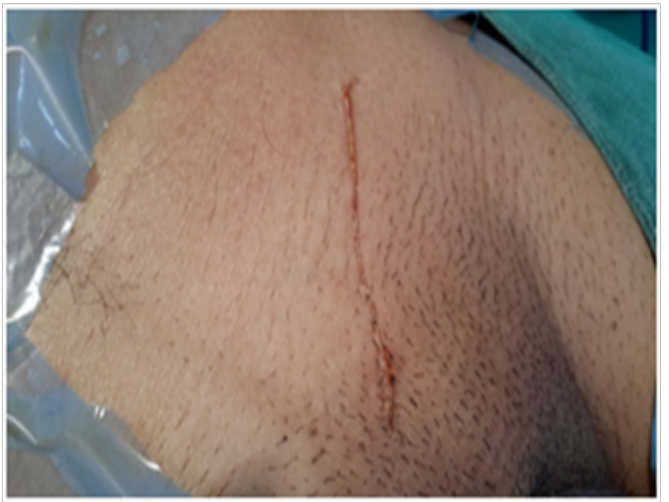

Figure 2 Left inguinal incision for inguinal hernia repair. 


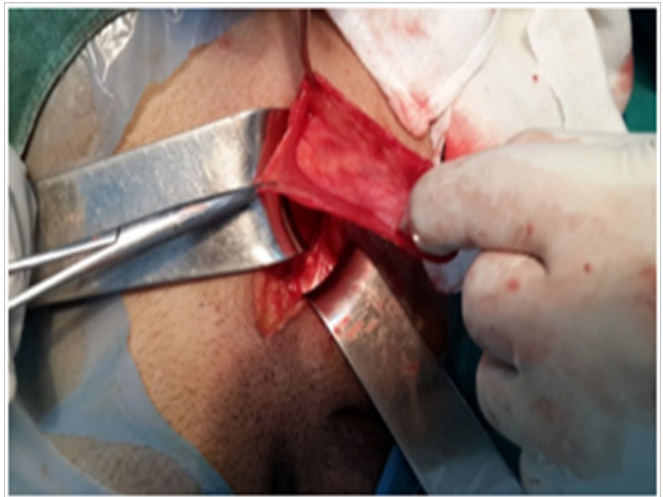

Figure 3 Hernial sac.

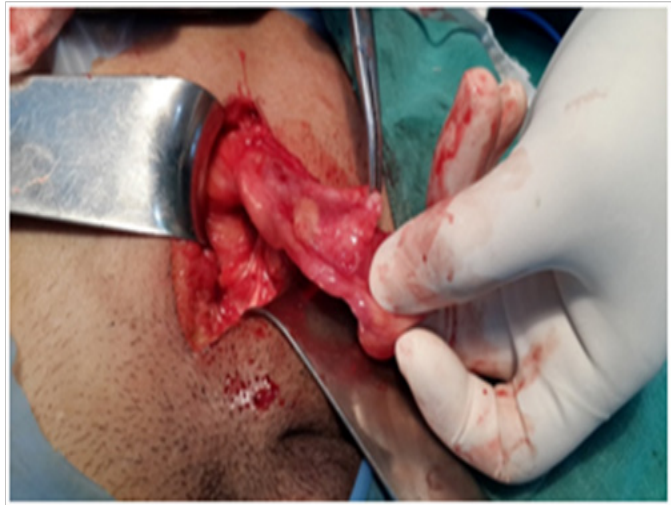

Figure 4 Epididymis and cord structures.

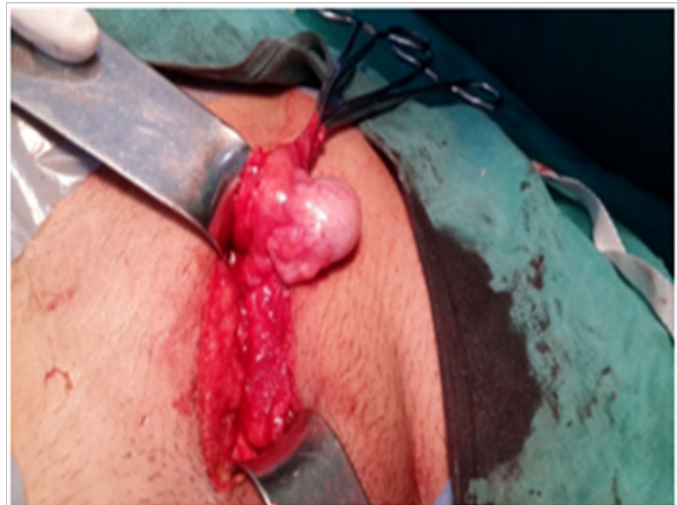

Figure $\mathbf{5}$ The left testis is visible coming out of the internal inguinal ring.

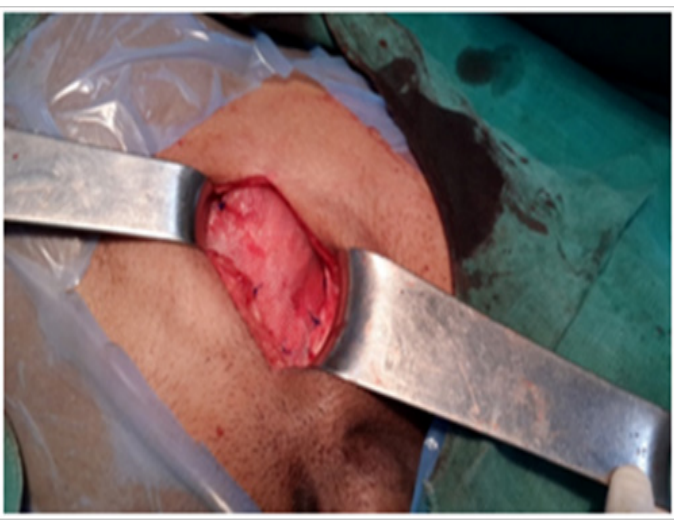

Figure 6 Prolene mesh fixed with prolene sutures.

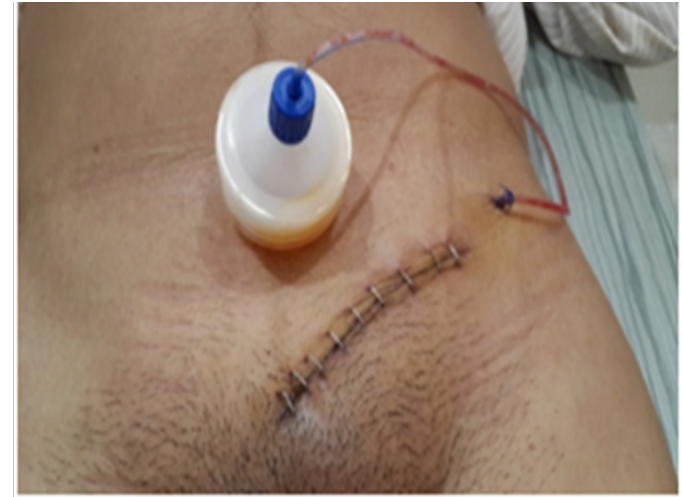

Figure 7 Skin closed with suction drainage.

\section{Discussion}

This was an extremely rare case of cryptorchidism revealed in an adult male patient. The patient remained asymptomatic for 60years. Most of the studies have concluded that there is a direct correlation between how long the testis was subjected to a cryptorchid position and testicular germ cell tumor (TGCT) incidence. The recommended age of surgical correction is before the age of 2years. In our case, we did not find a correlation between the time of surgery and the risk of TGCT. Histopathological examination confirmed a normal testis and the presence of leydig cells and the seminiferous tubule without testicular germ cell tumor (TGCT). An undescended testicle, sometimes called a cryptorchid testicle, can be found in 3\% of the term newborns and $0.5-1.0 \%$ of adults. ${ }^{1}$ Cryptorchidism is more commonly seen in premature babies and associated with genetic disorders in $10 \%$ of the cases. The common causes of cryptorchidism include premature birth, spina bifida, hormonal disorders, testicular absence and/or, retractile testes. Jensen MS et al concluded that smoking more than 10 cigarettes a day during pregnancy increased the risk of cryptorchidism. Jensen et al., ${ }^{2} \&$ Kaftanovskaya et al., ${ }^{3}$ concluded that the second inguino-scrotal stage of testicular descent is clearly androgen-dependent. ${ }^{3}$ The diagnosis of cryptorchidism is made by physical examination. The diagnosis of cryptorchidism should be considered when non-palpable testis and inguinal hernia are present, although, each patient might experience the symptoms differently. Nonetheless, for inguinal hernia, the clinical presentation varies, depending on the contents of the hernial sac and the degree of herniation. Because of its varied presentation, clinical examination is often inconclusive. ${ }^{4}$ In our case, the diagnosis of cryptorchidism was not arrived-at before carrying-out the surgical procedure as ultrasound abdomen was normal in the pre-operative period. The correct diagnosis of inguinal hernia is usually made during an inguinal hernia repair, although ultrasonography (USG) and computerized tomography (CT) have been used to identify an inguinal hernia. ${ }^{5}$ Laparoscopy is useful for both diagnosis and treatment of atypical inguinal hernias. ${ }^{6,7}$ The major complications of unrepaired cryptorchidism mainly include testicular cancers, inguinal hernia, testicular torsion and infertility. The testicles begin to lose the process of spermatogenesis if they are not in the scrotum possibly because of variations in temperature and other conducive environmental factors. This process explains the link between cryptorchidism and infertility. Very little is known, however, about the link between cryptorchidism and testicular germ cell tumor (TGCT) genesis. In our case, despite the age, histopathological examination confirmed the absence of tumor. Because the incidence of testicular cancer generally increases in cryptorchid testes, longterm follow-ups become mandatory. Usually, cryptorchidism resolves 
without any intervention before the age of 6months. Surgical repair for cryptorchidism is carried-out if the testicles have not descended till this age and surgical intervention becomes mandatory. The prognosis of cryptorchid testes is related to the precocity of the management. Most studies have concluded that there is a direct correlation between how long the testis was subjected to a cryptorchid position and the testicular germ cell tumor (TGCT) incidence. Pettersson et al., ${ }^{8}$ demonstrated that individuals who had corrective surgery after the age of $13 \mathrm{had}$ an incidence rate of $5.4 \%$ whereas those who were treated before had an incidence rate of $2.23 \%$. Pettersson et al., ${ }^{8}$ In our case, we did not find a correlation between the time of surgery and risk of TGCT incidence. As far as our case was concerned, histopathological examination confirmed a normal testis and the presence of leydig cells and the seminiferous tubule without testicular germ cell tumor (TGCT). The treatment of cryptorchidism improves the risks of infertility and gonadal neoplasia. Surgical repair for cryptorchidism results in an early detection of an eventual tumor. It is difficult to understand the surgical anatomy of inguinal hernias but once the surgical exploration is performed, surgical repair is uneventful. It is controversial whether a contralateral orchidopexy is needed. Furthermore, the incidence of testicular cancer generally increases in fixed testes. ${ }^{9}$ In our case, the contralateral orchidopexy was not performed. An alternative therapeutic option is hormonal treatment with a series of injections of HCG (Human Chorionic Gonadotropin) which stimulate the testis. Kjaer et al. ${ }^{10}$ concluded that there was no age dependency observed of the HCG treatment effects though the position of the testis before treatment influenced the success rate. ${ }^{10}$ This treatment is recommended if the testis is very close to the scrotum. Our case was notable because of the unusual presentation of cryptorchid testis as an incarcerated inguinal hernia at the age of $60 y e a r s$ and the patient remained asymptomatic for 60years. To our knowledge, the present case represents the first case of cryptorchid testis revealed at the age of 60years with an inguinal hernia.

\section{Conclusion}

The surgeon must always be alert to the possibility of cryptorchid testis during a surgical exploration of an inguinal hernia. In suspected cases, ultrasonography (USG) and computerized tomography (CT) as well as laparoscopic evaluation may be helpful in diagnosing this atypical inguinal hernia before carrying-out the requisite surgical procedure.

\section{Acknowledgements}

None.

\section{Conflict of interest}

The author declares no conflict of interest.

\section{References}

1. Longui CA. Cryptorchidism: Diagnosis and treatment. Arq Bras Endocrinol Metabol. 2005;49:165-171.

2. Jensen MS, Toft G, Thulstrup AM, et al. Cryptorchidism according to maternal gestational smoking. Epidemiol. 2007;18(2):220-225.

3. Kaftanovskaya EM, Huang Z, Barbara AM, et al. Cryptorchidism in mice with an androgen receptor ablation in gubernaculum testis. Mol Endocrinol. 2012;26(4):598-607.

4. Kassir R, Tarantinob E, Lacheze R, et al. Management of Spigelian hernia caused by necrobiotic fibroma of the uterus in a pregnant woman. Int J Surg Case Rep. 2013;4:1176-1178.

5. Tasian GE, Copp HL, Baskin LS. Diagnostic imaging in cryptorchidism: Utility, indications and effectiveness. J Pediatr Surg. 2011;46(12):24062413.

6. Mehendale VG, Shenoy SN, Shah RS, et al. Laparoscopic management of impalpable undescended testes: 20years' experience. J Minim Access Surg. 2013;9(4):149-153.

7. Kassir R, Bourbon M. Management of the Aymand's Hernia in laparoscopy. J Curr Surg. 2013;3:92-94.

8. Pettersson A, Richiardi L, Nordenskjold A, et al. Age at surgery for undescended testis and risk of testicular cancer. $N$ Engl $\mathrm{J} \mathrm{Med}$. 2007;356(18):1835-1841.

9. Walsh TJ, Dal'Eral MA, Croughan MS, et al. Pre-pubertal orchiopexy for cryptorchidism may be associated with lower risk of testicular cancer. J Urol. 2007;178(4):1440-1446.

10. Kjaer S, Mikines KJ. HCG in the treatment of cryptorchidism: The effect of age and position of the testis. Ugeskr Laeger. 2006;168(14):14481451 . 